\title{
The Italian SurveY on carDiac rEhabilitation - 2008 (ISYDE-2008): a snapshot of current cardiac rehabilitation programmes and provides in Italy Part 2: ISYDE-2008 investigators and directory of italian cardiac rehabilitation centers
}

\section{ISYDE-2008: II Survey 2008 sulla Cardiologia Riabilitativa Italiana: una istantanea delle strutture e dei programmi di Cardiologia Riabilitativa in Italia \\ Parte 2: Elenco dei centri e degli investigatori dei centri italiani di cardiologia riabilitativa}

\author{
Roberto Tramarin1, Stefania De Feo2, Marco Ambrosetti3, Raffaele Griffo4, \\ Franco Maslowsky ${ }^{1}$, Paola Vaghi5 \\ on behalf of the ISYDE-2008 Investigators of the Italian Society \\ of Cardiac Rehabilitation and Prevention (GICR) 6
}

\footnotetext{
Monaldi Arch Chest Dis 2008; 70: 1-5.

1 Cardiac Rehabilitation Unit, Fondazione Europea per la Ricerca Biomedica, Cernusco S/N (MI).

2 U.O. Cardiology, Casa di Cura Polispecialistica "Dr. Pederzoli", Peschiera del Garda (VR).

3 Cardiovascular Rehabilitation Unit, "Le Terrazze" Clinic, Cunardo (VA).

4 Cardiology Department, La Colletta Hospital, Arenzano (GE).

5 Gruppo Italiano di Cardiologia Riabilitativa e Preventiva, Genova (I).

6 The ISYDE-2008 Investigators are listed in the Appendix .
}

From January 28th to February 10th, 2008 the Italian Society of Cardiac Rehabilitation and Prevention (GICR) conducted a national web-based survey aimed to provide specific and updated information on organization and core components of cardiac rehabilitation $(\mathrm{CR})$ in Italy, and to describe, both in residential and out-patient settings, indications for CR, clinical characteristics of referred patients, risk profile, performed diagnostic procedures, exercise and educational programme, treatments and arrangements for the follow-up $[1,2]$.

The ISYDE-2008 is a multicenter, longitudinal, prospective observation study whose design was previously described $[1,2]$.

\section{Participating Centres}

All 144 CR units of the 2004 inventory were invited to join the survey. In addition, all regional coordinators of the GICR were asked to update the list of CR units indicating new or potentially active centers.
Out of 208 facilities supposed to deliver CR programmes and invited to participate into the survey, 18 were shown actually "virtual". Twenty-five (13\%) of 190 Italian CR units, did not join the study, and 22 were still not active at the moment of the survey (table 1).

The list of 165 participating centres and the names of responsible or referent physicians are reported in Appendix and the up-date directory of all Italian Cardiac Rehabilitation Centres of the GICR network has been published on the website of the GICR http://www.gicr.it.

The regional distribution of CR units is reported in Table 1. According to these data in the last 4 years 21 new CR centres were created in Italy $(+15 \%)$.

\section{Study population}

In coherence to expectations of the ISYDE-2008 scientific board, the participating centres recruited 2281 consecutive patients discharged from rehabilitation program between the $28^{\text {th }}$ January and the $10^{\text {th }}$ February 2008. 


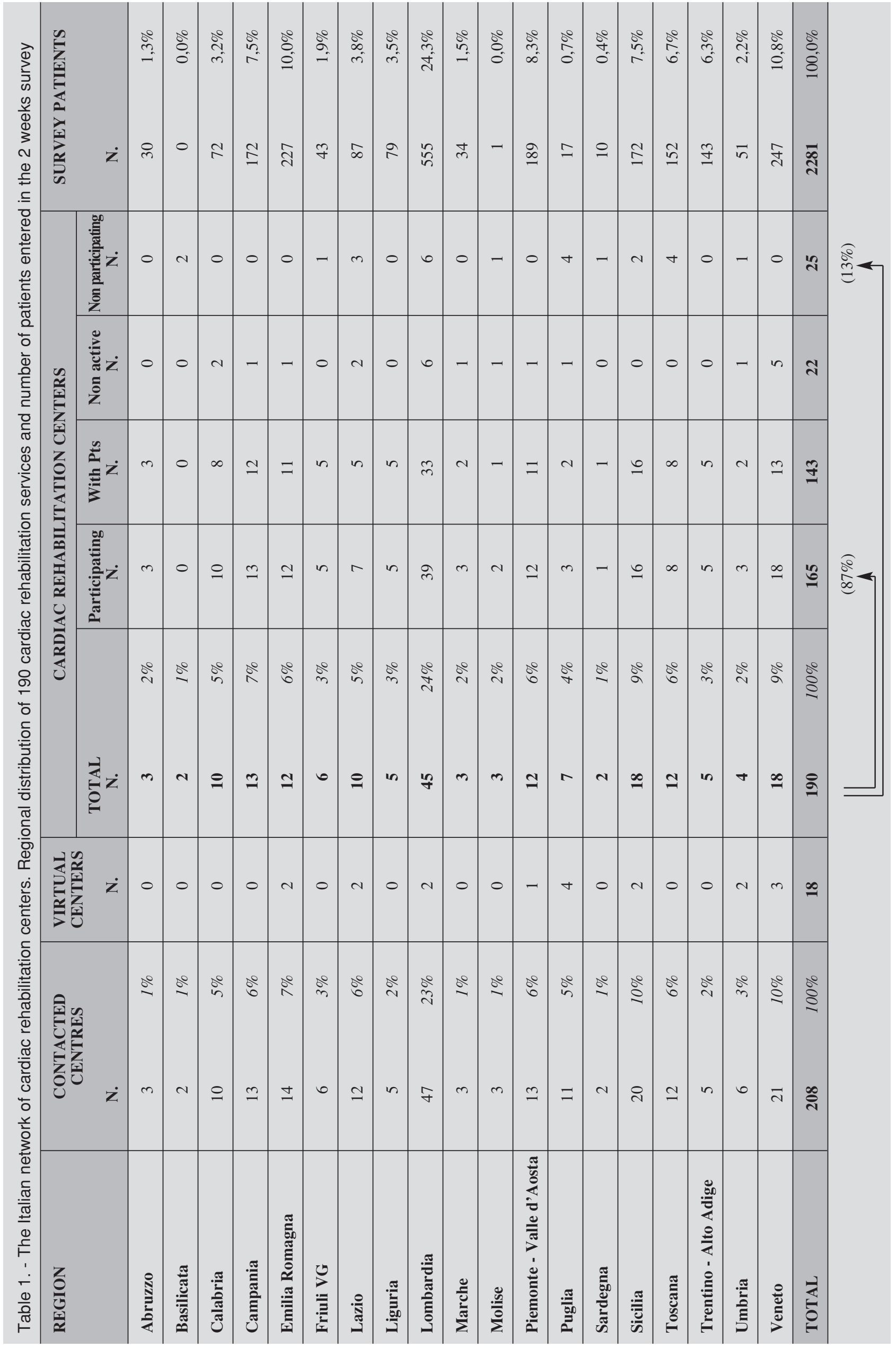


The analysis and evaluation of data is ongoing and the final results will be presented at the National GICR Congress in Ostuni, October 23-25, 2008.

The broad participation of CR centres throughout Italy and the dimension of surveyed population proved to represent the real, pragmatic rehabilitation world and clinical profile of patients referred to these programmes.

The detailed snapshot of CR in Italy, provided by ISYDE-2008 will give an insight in the level of implementation of contemporary guidelines and recommendation of $\mathrm{CR}$ and secondary prevention.

Appendix

\section{Scientific Board}

R. Tramarin (Cernusco S/N - MI), R. Griffo (Arenzano - GE), C. Riccio (Caserta), R. Carlon (Cittadella - PD), A. Castello (Palermo), M. Ferratini (Milano), C. Schweiger (Milano), C. Chieffo (Napoli), C. Vigorito (Napoli)

\section{Writing Committee}

R. Tramarin (Cernusco S/N - MI), M. Ambrosetti (Cunardo - VA), S. De Feo (Peschiera del Garda - VR), R. Griffo (Arenzano - GE)

\section{Coordination}

M. Ambrosetti (Cunardo - VA), S. De Feo (Peschiera del Garda - VR), T. Diaco (Rivolta d'Adda - CR). Delegati Regionali del Gruppo Italiano di Cardiologia Riabilitativa e Preventiva: Abruzzo: C. Ciglia (Chieti), Basilicata: A. Lapolla (Policoro - MT), Calabria: F. Pietropaolo (Soriano Calabro - VV), Campania: G. Furgi (Telese Terme - BN), Friuli Venezia Giulia: G. Martin (Sacile - PN), Emilia Romagna: P. Semprini (Rimini), Lazio: A. Avallone (Roma), Liguria: D. Bertoli (Sarzana - SP), Lombardia: T. Diaco (Rivolta d'Adda - CR), Marche: R. Belardinelli (Ancona), Piemonte: F. Soffiantino (Lanzo Torinese - TO), Puglia: D. Scrutinio (Cassano Murge - BA), Sardegna: P. Maxia (Cagliari), Sicilia: R. Coco (Catania), Toscana: C. Giustarini (Volterra - PI), Umbria: P. Anniboletti (Perugia), Veneto: G. Favretto (Motta di Livenza - TV).

\section{Data and CRF management}

P. Vaghi (Saronno), P. Calisi (Arenzano - GE), C. Chiavetta (Genova), Aristea SpA (Genova), Segno \& Forma SpA (Milano)

\section{ISYDE-2008 Investigators}

Abruzzo: Leonello Guarracini - Presidio Ospedaliero Tagliacozzo - Tagliacozzo; Carlo Ciglia - Casa di Cura Villa Pini D’Abruzzo - Chieti; Giorgio - Marcellini - Presidio Ospedaliero S. Omero - S. Omero. Calabria: M. Iannopollo - Presidio Ospedaliero Siderno - USL 9 Locri (RC) - Siderno; Gennarino Borrello - Azienda Ospedaliera Mater Domini - Catanzaro; Milena Matta - Presidio Ospedaliero di Cetraro - Cetraro; N. Peccerillo - Ospedale di Mormanno - ASL 2 Castrovillari - Mormanno; Franco Boncompagni - Ospedale dell'Annunziata - Cosenza; Achil- le Amici - Casa di Cura "San Francesco" - Mendicino (CS) - Mendicino; Francesco Pietropaolo - Presidio Ospedaliero - Soriano Calabro; Emidio Feraco INRCA (IRCCS) - Cosenza; G. Pulitano - Presidio Ospedaliero Policlinico Madonna della Consolazione - Reggio Calabria; Ermete Tripodi - ASP 5 Regione Calabria - Scilla. Campania: Giuseppe Furgi - IRCCS Fondazione Salvatore Maugeri - Telese Terme; Carmelo Chieffo - Casa di Cura - Napoli; Soccorso Capomolla - Presidio Ospedaliero Polispecialistico Don Gnocchi - Sant'Angelo dei Lombardi; Paolo Golino - Azienda Ospedaliera di Caserta - Dipartimento di Cardiologia - Caserta; Gennaro Parrilli - Campolongo Hospital Spa Casa di Cura - Eboli; Antonio Di Patria - Casa di Cura Villa delle Magnolie - Castel Morrone; Claudio Di Gioia - Casa di Cura Privata Villa Margherita, Istituto S. Giuseppe Moscati - Benevento; Domenico Miceli - Azienda Ospedaliera V. Monaldi - Napoli; Carlo Vigorito Azienda Universitaria Policlinico - Università di Napoli Federico II - Napoli; Silvio Perrotta - ASL NA1 - Napoli; Alessandro Rubino - Azienda S. G. Moscati - Avellino; Vincenzo Aulitto - Centro Serapide SpA - Pozzuoli; Gerardo Riccio - Ospedale di Nocera - Nocera Inferiore. Emilia Romagna: Luciano Codec - Azienda Universitaria Ospedale S. Anna - Ferrara; Paolo Coruzzi - Fondazione Don Carlo Gnocchi - Onlus - Parma; Enrico Violi Azienda Ospedaliera di Reggio Emilia - Castelnovo Monti; Massimo Nardini - Ospedale Regina Margherita - Castelfranco Emilia; Stefano Urbinati Azienda USL di Bologna - Bologna; Sylvia Dcruz Casa di Cura Privata ad Indirizzo Riabilitativo San Giacomo srl - Ponte dell'Olio; Pierluigi Semprini Ospedale Infermi Rimini - Rimini; Massimo Piepoli - Azienda Unità Sanitaria Locale di Piacenza Piacenza; Alessandro Fucili - Ospedale Sant'Anna Ferrara; Massimo Cerulli - Ospedale Privato Accreditato Villa Pineta - Gaiato di Pavullo N/F; Bini Roberto - Azienda USL Cesena - Cesenatico; Guido Balestra - Villa Maria Cecilia Hospital - Cotignola. Friuli Venezia Giulia: Dulio Tuniz - IMFR Udine ASS N. 4 - Udine; Giovanni Martin - Azienda Ospedaliera Santa Maria degli Angeli Pordenone - Sacile; Pierpaolo Gori - Casa di Cura Pineta del Carso Aurisina; Roberto Marini - ASS N. 2 Isontina - Gorizia; Tullio Morgera - Ospedale San Polo - Monfalcone. Lazio: Franco Di Mario - Istituto Clinico Riabilitativo Villa delle Querce - Nemi; Maurizio Volterrani - IRCCS San Raffaele Pisana - Roma; Alfonso Galati - Presidio Ospedaliero Santo Spirito, Presidio Villa Betania, ASL Roma E - Roma; Alessandro Salustri - Policlinico Luigi Di Liegro - Roma; Anna Patrizia Jesi - Presidio Ospedaliero - Roma; Augusto Lacch - Azienda Ospedaliero San Camillo - Forlanini - Roma; Francesco Loperfido - Complesso Integrato Columbus. Università Cattolica del Sacro Cuore - Roma. Liguria: Luca - Corsiglia - Istituto Cardiovascolare Camogli - Ruta di Camogli; Raffaele Griffo - ASL 3 "Genovese" - Arenzano; Paolo Pantaleo - Struttura Ospedaliera Privata Accreditata Villa Azzurra - Rapallo; Guido Gigli - Regione Liguria ASL 4 - Centro Regionale di Riabilitazione Cardiologica - Sestri Levante; Daniele Bertoli - ASL 5 Spezzino - Sarzana. Lombardia: Diego Marchesi - Clinica Domus Salutis Ancelle della 
Carità - Brescia; Oreste Febo - IRCCS Fondazione Salvatore Maugeri - Montescano; Franco Cobelli IRCCS Fondazione Salvatore Maugeri Pavia - Pavia; Claudio Malinverni - Azienda Ospedaliera "Bolognini" - Seriate; Maurizio Ferratini - Fondazione Don Gnocchi Onlus - Milano; Norman Jones Ospedale Trabattoni Ronzoni - Seregno; Claudio Anzà - Multimedica Holding - Presidio Ospedaliero di Castellanza - Castellanza; R. Frizzelli - Azienda Ospedaliera C. Poma di Mantova - Bozzolo; Paolo Gei - Spedali Civili di Brescia - Gardone Riviera; Flavio Acquistapace - Policlinico di Monza - Monza; Roberto Pedretti - IRCCS Fondazione Salvatore Maugeri - Tradate; Roberto Tramarin - Fondazione Europea di Ricerca Biomedica - Onlus - Cernusco S/N- Milano; Stefano Carugo - Centro di Cardiologia Riabilitativa - Pio Albergo Trivulzio - Milano; Gabriella Malfatto - Istituto Auxologico Italiano IRCCS - Milano; Tommaso Diaco - Azienda Ospedale Maggiore di Crema - Rivolta D’Adda; Marco Ambrosetti - Clinica Le Terrazze - Cunardo; Carlo Meloni - Ospedale San Raffaele IRCCS U.O. Riabilitazione Cardiaca - Milano; Amerigo Giordano IRCCS Fondazione Salvatore Maugeri - Lumezzane; Alberto Schizzarotto - Azienda Ospedaliera S. Antonio Abate di Gallarate - Somma Lombardo; Renzo Zanettini - Azienda Ospedaliera Istituti Clinici di Perfezionamento - Milano; Roberto Bosco Ospedale Romano di Lombardia - Romano di Lombardia; Giuseppe Occhi - Azienda Ospedaliera della Valtellina e Valchiavenna - Sondalo; Sfefano Aglieri - Istituto Clinico Humanitas - Rozzano; Giuseppe Caprioli - Humanitas - Cliniche Gavazzeni SpA Bergamo; Nicola Cuocina - Azienda Ospedaliera Ospedali Riuniti di Bergamo - Bergamo; S. Salvetti - Centro di Cardiologia Riabilitativa di Cesano Boscone - Casa di Cura Ambrosiana - Cesano Boscone; Cinzia - Savonelli - Cof Lanzo Hospital S.p.A. Clinica Ortopedica e Fisiatrica. - Lanzo D'Intelvi; Carlo Schweiger - Azienda G. Salvini Garbagnate Milanese - Rho; Giovanni Corda - Azienda Ospedaliera ed Universitaria: Ospedale di Circolo ed Università dell'Insubria - Varese - Cuasso Al Monte; Claudia Meinecke - Azienda Istituti Ospitalieri di Cremona - Cremona; Castiglioni - ASL Prov. Milano - Presidio Ospedaliero Cassano D'Adda - Cassano D'Adda; Ravizza Pierfranco - Azienda Ospedaliera Ospedale di Lecco - Merate; Paolo Giani - Fondazione Europea di Ricerca Biomedica - Trescore Balneario; F. Robustelli - Ospedale di Morbegno ASL Provincia di Sondrio - Morbegno; Giuseppe Gullace - Azienda Provinciale Ospedale di Lecco Lecco; F. Passoni - Azienda Ospedaliera di Legnano - Cuggiono; Maria Vittoria Barelli - Centro Fisioterapico Riabilitativo Club Francesco Conti - Milano; Italo Richichi - Ospedale San Matteo U.O. di Riabilitazione - Belgioioso; Angelo Rossi - Casa di Cura Villa Esperia - Salice Terme; Politi - Ospedale Generale di Zona - Gravedona. Marche: Romualdo Belardinelli - Azienda Ospedaliero-Universitaria Umberto I - Ancona; Casa di Cura Villa Serena - Jesi; Mauro Persico - ASUR Marche Zona Territoriale 12, San Benedetto del Tronto - Area Vasta N. 5 San Benedetto del Tronto. Piemonte e Valle d'Aosta: Francesco Alessandrini - Università Cattolica del Sacro Cuore - Facoltà di Medicina e Chirurgia;
Pantaleo Giannuzzi - IRCCS Fondazione Salvatore Maugeri, Clinica del Lavoro e della Riabilitazione Veruno; Gianmauro Mazzucco - Casa di Cura Villa Serena - Centro medico di riabilitazione cardiologica e pneumologica - Piossasco; Enzo Bosimini - Casa di Cura Major - Sede Distaccata IRCCS Fondazione Maugeri Veruno - Torino; Giuseppe Riva - Casa di Cura Riabilitativa Stella del Mattino - Boves; Francesco Soffiantino - Presidio Ospedaliero di Lanzo - Lanzo Torinese; Nicola Massobrio - Ospedale Evangelico Valdese di Torino - Torino; Luca Gondoni - Unità Operativa ad indirizzo Cardiologico - IRCCS Istituto Auxologico Italiano - Piancavallo di Oggebbio; Franco Aina - Azienda Ospedaliero-Universitaria Maggiore della Carità di Novara - Novara; Biagio Ingignoli - Ospedale Ss Trinità Fossano; Maria Vincenza Cal - Ospedale di Acqui Terme - Acqui Terme; Antonio - Carpagnano - U.O. Dipartimentale di Cardiologia Riabilitativa; Aillon Costante - Azienda USL Valle D'Aosta - Ospedale Regionale "Umberto Parini" - Aosta. Puglie: Domenico Scrutinio - IRCCS Fondazione Salvatore Maugeri - Cassano Murge; Massimo Villella IRCCS Casa Sollievo della Sofferenza - San Giovanni Rotondo; Concetta La Rosa - Casa di Cura Villa Verde snc - Taranto. Sardegna: Luigi Sannia ASL Sassari - Sassari. Sicilia: Paolo Barbanto - Presidio Ospedaliero M. Ascoli - S. Tommaselli, Azienda Ospedaliera -Catania; Antonio Castello - Ospedale Buccheri La Ferla Fatebenefratelli - Palermo; Rosario Coco - Azienda Ospedaliera Cannizzaro Dipartimento Cardiovascolare - Catania; Renato Rametta - Azienda Ospedaliera "San Giovanni di Dio" - Agrigento; Daniele Albanese - Unità di Valutazione Funzionale e Riabilitazione del Cardiopatico Palermo; Antonio Circo - Casa di Cura Carmide Villa l'Ulivo - Catania; Rosaria Raciti - Centro Cuore Morgagni - Pedara; Luigi Scarnato - Casa di Cura Regina Pacis - San Cataldo; Flavia Dispensa - Fondazione Istituto San Raffaele Giglio - Cefalù; Leondardo La Spina - Centro Ionico di Cardiologia - Riposto; Alfio Stuto - Star For Life c/o Poliambulatorio di Cardiologia Dr. Armaro Biagio sas - Siracusa; Calogero Vasco - Azienda Ospedaliera Umberto I Enna; Salvatore Gibiino - Diagnostica Cardiovascolare del Dr. S. Gibiino \& C. snc. - Catania; Michele Gabriele - Ospedale di Mazara Del Vallo - Mazara Del Vallo; Claudio Dispensieri - Azienda Ospedaliera Piemonte - Messina; Vittorio Carini - Casa di $\mathrm{Cu}$ ra Riabilitativa "Villa Sofia" - Acireale. Toscana: Michele Provvidenza - ASL 12 Versilia - Presidio Ospedaliero di Viareggio - Lido di Camaiore; Alessandro Scalzini - ASL 6 Livorno - Presidio Ospedaliero di Cecina - Cecina; Carlo Giustarini - Auxilium Vitae Volterra SpA - Volterra; Claudio Macchi Fondazione Don Gnocchi Onlus, IRCCS - Impruneta; Francesco Fattirolli - Azienda Ospedaliero Universitaria Careggi - Firenze; Mauro Vannucci - IFCA Villa Ulivella e Glicini - Firenze; Luciana Iacopetti - ASL 3 Pistoia - Presidio Ospedaliero Pescia Pescia; Mario Cordoni - ASL 6 Livorno - Presidio Ospedaliero di Piombino - Piombino. Trentino e Alto Adige: Ferrario Gabriele - Casa di Cura Eremo - Arco; Renato Santoni - Ospedale Classificato San Pancrazio - Arco; Walter Pitscheider - Centro di Riabilitazione del Cardiopatico - Salus Center - Bol- 
zano; Roberto Bettini - Ospedale di Trento - Mezzolombardo; Daniela Girardini - S. Maria del Carmine - Rovereto - Centro di Riabilitazione Cardiologica Ospedale di Ala - Ala. Umbria: Sara Mandorla ASL 1 - Gualdo Tadino - Gualdo Tadino; Fabrizio Anniboletti - ASL 2 Servizio di Cardiologia Ex Ospedale Grocco Perugia - Perugia; Federico Patriarchi - U.O. Cardiologia Ospedale di Foligno Nocera Umbra. Veneto: Giuseppe - Favretto - Ospedale Riabilitativo di Alta Specializzazione SpA Motta di Livenza; Pier Luigi Baroni - Casa di Cura Villa Garda - Garda; Fede Bordin - ASL N. 4 - Presidio Ospedaliero di Thiene - Thiene; Pietro Biondi - ULSS 8 - Montebelluna; Fabio Bellotto - Istituto Riabilitativo - Cortina D'Ampezzo; Albino Zanocco - Azienda Ospedaliera - Mirano; Andrea Ponchia ULSS 16 - Padova; Enzo Mantovani - Azienda ULSS 15 - Camposampiero; E. Li Greci - Ospedale Don L. Chiarenzi - AALSS 21 Legnago (Verona) Zevio; Roberto Carlon - Azienda ALSS 15 Presidio Ospedaliero di Cittadella - Cittadella; Leopoldo Celegon - U.O. di Cardiologia - Riabilitazione Cardiologica - Castelfranco Veneto; Stefano Baracchi Ospedale al Mare Lido di Venezia - Lido di Venezia; G. Pizzolato - AUSSL N. 10 - San Donà di Piave; Lorenzo Peroni - Policlinico San Marco - Mestre; Enzo Apolloni - Ospedale di Asiago - Asiago; - Casa di Cura Privata Villa Margherita Srl - Arcugnano; M. Guarniero - Ospedale di Feltre - Feltre; Loris Roncon - Ospedale di Rovigo - Rovigo.
The Directory of Italian Cardiac Rehabilitation Centres is now available on the GICR website http://www.gicr.it

\section{Organization}

The Italian Working Group on Cardiac Rehabilitation (GICR) has the full responsibility of the ISYDE-2008, not only for the formulation, but also for the overall conduction of the survey, including onsite monitoring, interpretation and utilization of the results. Administration location: Aristea International srl, Salita Santa Caterina 4/7 - 16123 Genova.

\section{References}

1. Tramarin R, De Feo S, Ambrosetti M, Griffo R, Maslowsky F, Vaghi P, on behalf of the ISYDE 2008 Investigators of the Italian Society of Cardiac Rehabilitation and Prevention (GICR). ISYDE-2008 Study presentation: The Italian SurveY on carDiac rEhabilitation: a snapshot of current cardiac rehabilitation, programmes and provides in Italy. Monaldi Arch Chest Dis 2007; 68: 193-198.

2. Tramarin R, Ambrosetti M, De Feo S, Griffo R, Maslowsky F, Vaghi P, a nome dei Ricercatori del Progetto ISYDE2008 del Gruppo Italiano di Cardiologia Riabilitativa e Preventiva (GICR). Il progetto ISYDE-2008 (Italian SurveY on carDiac rEhabilitation): strutture, rete, organizzazione e programmi di cardiologia riabilitativa in Italia. G Ital Cardiol 2008; 9 (6): in press. 